\title{
Peculiarities of the Structure and Function of AMPA Receptors Revealed from the Action of Channel Blockers
}

\author{
T. B. Tikhonova, D. B. Tikhonov, and L. G. Magazanik \\ Sechenov Institute of Evolutionary Physiology and Biochemistry, Russian Academy of Sciences, St. Petersburg; \\ e-mail: denistikhonov2002@yahoo.com
}

DOI: $10.1134 / \mathrm{S} 1990747809030489$

Dicationic derivatives of adamantine, phenylcyclohexyl and diphenyl are potent channel blockers of AMPA type ionotropic glutamate receptors. Our previous studies have revealed structural and molecular determinants of binding of AMPA receptor channel blockers. More detailed investigation of the mechanisms of the blocking action offers a clearer view of structure and activation mechanisms of the channels.

The following conclusions were made in the present study: (i) extracellular drug application results in the open channel block, indicating that the binding site is guarded by the activation gate of the channel; (ii) drug binding does not prevent channel closure and blockers get trapped in the closed channels; (iii) blockers can permeate though the open channel and dissociate from the closed channel into the cytoplasm; (iv) intracellular application of the drugs causes the block of open and closed channel conformations; (v) blockers bind to a single site in the channel independent on the side of application. Analysis of the whole set of the data using molecular and kinetic models demonstrates that the channel activation gate controls the access to the binding site from the external but not from the internal side of the membrane. It means that the selectivity filter of the AMPA receptor channel is permeable to organic compounds even in the closed state. This conclusion significantly contributes to the notion of the activation mechanisms of ionotropic glutamate receptors. 